\section{Adrenalectomía laparoscópica en un paciente con quiste broncogénico}

Briseño-Rentería JI, ${ }^{1}$ López-Verdugo JF, ${ }^{2}$ Estrada-Bujanos JM,${ }^{3}$ Camacho-Trejo VC, ${ }^{1}$ Castro-Zazueta S, ${ }^{1}$ Quintero-Badillo JE, ${ }^{1}$ Tejeda-Andrade CF, ${ }^{1}$ MoralesMondragón JC ${ }^{1}$

\begin{abstract}
Resumen
ANTECEDENTES: Los quistes broncogénicos son formaciones congénitas provenientes del desarrollo fetal anómalo del árbol traqueobronquial. El sitio más común es el tórax; sin embargo, se han reportado casos excepcionales en el abdomen, debido al retraso en la migración de estructuras abdominales durante la gestación.
\end{abstract}

CASO CLÍNICO: Paciente femenina de 53 años de edad, que acudió al servicio de Urgencias con dolor agudo en la fosa renal izquierda, sin reacción al tratamiento con analgésicos convencionales. La urotomografía computada evidenció la glándula adrenal con una masa de bordes bien definidos, de $7.1 \times 4.3 \times 6.0 \mathrm{~cm}$, sin realce con la administración del medio de contraste (densidad promedio de 37 UH), sugerente de un quiste con contenido denso. La resonancia magnética en secuencia T2 mostró una masa retroperitoneal adyacente a la parte interna del riñón, que desplazaba la glándula suprarrenal izquierda, de 38 x 67 mm de diámetro en corte axial. En secuencia T1 se comportó de manera hiperintensa, pero no se observó reforzamiento con el paso del medio de contraste, que también fue sugerente de un quiste. Se decidió la adrenalectomía laparoscópica izquierda, sin complicaciones transquirúrgicas y con sangrado estimado de 100 cc. Con el reporte histopatológico se estableció el diagnóstico de quiste bronquial subdiafragmático.

CONCLUSIÓN: Los pacientes con quistes broncogénicos suelen cursar asintomáticos y su diagnóstico es fortuito. El tratamiento retroperitoneal consiste en la extirpación quirúrgica. Los quistes broncogénicos deben considerarse en el diagnóstico diferencial de masas suprarrenales.

PALABRAS CLAVE: Quiste broncogénico; laparoscopia; glándula suprarrenal; adrenalectomía; quiste broncogénico retroperitoneal.

Rev Mex Urol. 2018 Jan-Feb;78(1):57-61.

\section{Laparoscopic adrenalectomy in a patient with bronchogenic cyst}

Briseño-Rentería JI, ${ }^{1}$ López-Verdugo JF, ${ }^{2}$ Estrada-Bujanos JM, ${ }^{3}$ Camacho-Trejo VC, ${ }^{1}$ Castro-Zazueta S, ${ }^{1}$ Quintero-Badillo JE, ${ }^{1}$ Tejeda-Andrade CF, ${ }^{1}$ MoralesMondragón JC ${ }^{1}$

\section{Abstract}

BACKGROUND: Bronchogenic cysts are congenital formations derived from anomalous fetal development of the tracheobronchial tree. The
${ }^{1}$ Urólogo, Fellowship en Endourología y Laparoscopia avanzada.

${ }^{2}$ Urólogo, director.

${ }^{3}$ Urólogo.

Instituto de Urología Robótica y Laparoscópica (IURL), Hospital San José, Tecnológico de Monterrey, Nuevo León, México.

Recibido: septiembre 2017

Aceptado: enero 2018

Correspondencia

Juan Iván Briseño Rentería

dr_briseno@hotmail.com

Este artículo debe citarse como

Briseño-Rentería JI, López-Verdugo JF, EstradaBujanos JM, ,Camacho-Trejo VC, Castro-Zazueta S, Quintero-Badillo JE, Tejeda-Andrade CF, MoralesMondragón JC. Adrenalectomía laparoscópica en un paciente con quiste broncogénico. Rev Mex Urol. 2018 ene-feb;78(1):57-61.

DOI: https://doi.org/10.24245/revmexurol.v78i1.1650 
most common location is the chest, but they have been reported in the abdomen, due to delay in the migration of abdominal structures during gestation

CLINICAL CASE: A 53-year-old woman came to the emergency service with acute pain in the left renal fossa that did not respond to conventional analgesics. Computed tomography urography revealed a $7.1 \times 4.3 \times 6.0-\mathrm{cm}$, non-enhancing mass (mean density of 37HU) with well-defined edges at the adrenal gland, suggestive of a cyst with dense content. Magnetic resonance imaging in the T2-weighted sequence showed a retroperitoneal mass adjacent to the internal part of the kidney, displacing the left adrenal gland. It measured $38 \times 67$ $\mathrm{mm}$ in diameter in the axial view. The T1-weighted sequence was hyperintense, but the lesion was non-enhancing, suggesting a cyst. Laparoscopic left adrenalectomy was performed with no intraoperative complications and an estimated blood loss of $100 \mathrm{cc}$. The histopathologic study diagnosis was sub-diaphragmatic bronchial cyst.

CONCLUSIONS: Patients with bronchogenic cysts tend to be asymptomatic and diagnosis is usually incidental. Retroperitoneal treatment is surgical removal. Bronchogenic cysts should be considered in the differential diagnosis of adrenal gland masses.

KEYWORDS: Bronchogenic cyst; Laparoscopy; Adrenal gland; Adrenalectomy; Retroperitoneal bronchogenic cyst.

\section{ANTECEDENTES}

Los quistes broncogénicos son formaciones congénitas provenientes del desarrollo fetal anómalo del árbol traqueobronquial. Los órganos del sistema respiratorio y el esófago tienen como origen común el intestino primitivo..$^{1-3}$ La localización definitiva o específica de estas malformaciones depende del estadio embriológico en el que se originan. Las anomalías tempranas aparecen en la tráquea, la carina o los bronquios principales, dando lugar a los quistes broncogénicos de mediastino, que generalmente se manifiestan como estructuras únicas y de tamaño variable (pueden medir menos de $1 \mathrm{~cm}$ y en ocasiones ocupan más de un compartimento mediastinal)., ${ }^{1,2}$

Los quistes broncogénicos suelen localizarse en el tórax; sin embargo, se han reportado casos en el abdomen, debido al retraso en la migración de estructuras abdominales durante la gestación, incluso detenidas por el diafragma, lo que origina este tipo de quistes, cuyo mecanismo exacto aún se desconoce. ${ }^{1,2,4,5}$

El diagnóstico diferencial se establece con adenoma suprarrenal, quiste mucinoso, teratomas, entre otros; no obstante, el diagnóstico certero resulta del análisis de la pieza quirúrgica. El acceso quirúrgico aún se discute, pues se desconoce su relación con tumores malignos. ${ }^{2,5}$

\section{CASO CLÍNICO}

Paciente femenina de 53 años de edad, con antecedente de hipertensión arterial diagnosticada 10 años atrás, actualmente en tratamiento con losartan-hidroclorotiazida ( 1 tableta cada $12 \mathrm{~h}$ por 
vía oral). Acudió al servicio de Urgencias con dolor agudo en la fosa renal izquierda, sin reacción al tratamiento con analgésicos convencionales.

En la exploración física se encontraron signos vitales estables; afebril, con giordano positivo, además de eritema y erupción herpética en el flanco izquierdo, sin relación con dolor referido por la paciente.

Los estudios de laboratorio reportaron: leucocitos $9 \times 10 / \mathrm{UL}$, eritrocitos $3.6 \times 10 / \mathrm{UL}$, hemoglobina $11 \mathrm{~g} / \mathrm{dL}$, hematocrito $32 \%$, plaquetas $340 \times 10 /$ UL, glucosa $110 \mathrm{mg} / \mathrm{dL}$, creatinina $0.8 \mathrm{mg} / \mathrm{dL}$, urea $30 \mathrm{mg} / \mathrm{dL}$, calcio $9 \mathrm{mg} / \mathrm{dL}$, albúmina $3.4 \mathrm{~g} /$ $\mathrm{dL}$, TGO $23 \mathrm{U} / \mathrm{L}$, TGP $34 \mathrm{U} / \mathrm{L}$, fosfatasa alcalina $103 \mathrm{U} / \mathrm{L}$, cortisol $15.80 \mathrm{mg} / \mathrm{dL}$, ACTH $31 \mathrm{pg} / \mathrm{mL}$ y metanefrinas en orina de 24 horas $<10 \mathrm{pg} / \mathrm{mL}$.

La urotomografía computada evidenció la glándula adrenal con masa de bordes bien definidos, de $7.1 \times 4.3 \times 6.0 \mathrm{~cm}$, sin realce con la administración del medio de contraste (densidad promedio de $37 \mathrm{UH}$ ), sugerente de un quiste con contenido denso (Figura 1).

La resonancia magnética en secuencia T2 mostró una masa retroperitoneal adyacente a

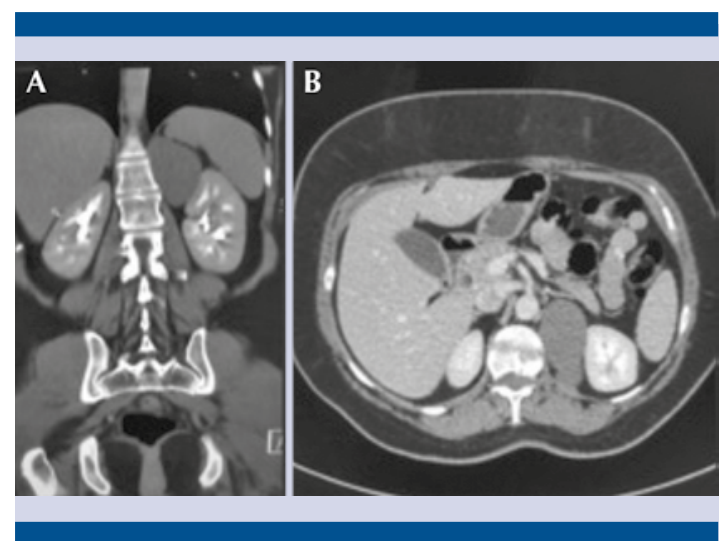

Figura 1. Urotomografía. Cortes coronal (A) y axial (B) que evidencian una masa de $7.1 \times 4.3 \times 6.0 \mathrm{~cm}$ en la glándula suprarrenal. la parte interna del riñón, que desplazaba la glándula suprarrenal izquierda, de $38 \times 67 \mathrm{~mm}$ de diámetro en corte axial. En secuencia T1 se comportó de manera hiperintensa, pero no se observó reforzamiento con el paso del medio de contraste, que también fue sugerente de un quiste (Figura 2).

La paciente tuvo mala evolución, persistió con dolor constante, pero disminuía con la administración de medicamentos. Por tal motivo se decidió efectuar adrenalectomía laparoscópica izquierda (Figura 3); la intervención se llevó a

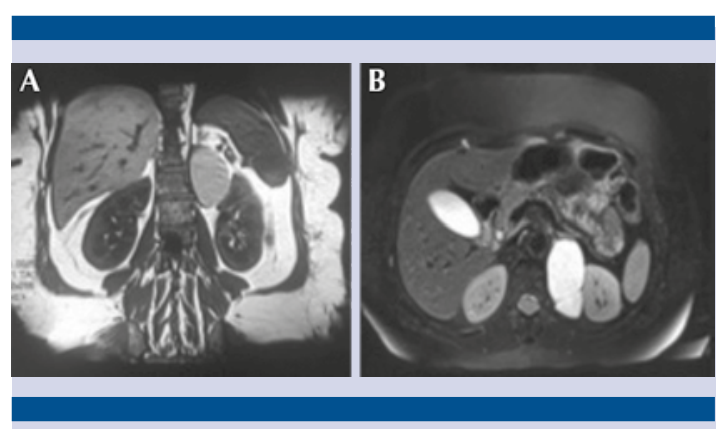

Figura 2. Resonancia magnética. Cortes coronal (A) y axial (B), este último con imagen hiperintensa, pero sin reforzamiento con el paso del medio de contraste.

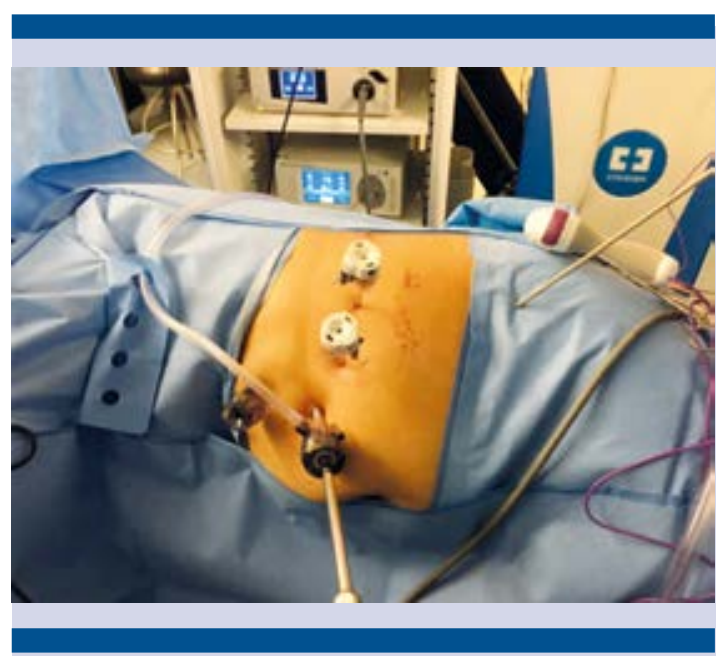

Figura 3. Posición del paciente y colocación de los trocares para efectuar la adrenalectomía. 
cabo en 70 minutos, sin complicaciones transquirúrgicas y con sangrado estimado de 100 cc.

El reporte de histopatología fue: pieza de adrenalectomía izquierda de $5.4 \times 3.4 \times 0.5 \mathrm{~cm}$, con quiste dependiente de la glándula adrenal de $7.0 \times 5.0 \times 4.0 \mathrm{~cm}$, separado por una membrana translúcida. Los cortes mostraron revestimiento epitelial respiratorio, pared con músculo liso y cartílago hialino; la glándula suprarrenal no evidenció alteraciones. El diagnóstico final fue quiste bronquial subdiafragmático (Figura 4).

\section{DISCUSIÓN}

En la bibliografía revisada a nuestro alcance se encontró que en 1953 Miller y sus colaboradores reportaron el primer caso de un quiste broncogénico retroperitoneal. ${ }^{4}$ Estos tumores suelen aparecer en el tórax, por lo que su localización retroperitoneal es extremadamente rara. La región peripancreática y la glándula suprarrenal izquierda son las localizaciones más comunes de los quistes broncogénicos situados en el retroperitoneo, esta última fue la localización de nuestro paciente..$^{4,5}$

Respecto de las teorías de la patogénesis de los quistes broncogénicos retroperitoneales, Sumiyoshi y su grupo describieron la carencia en el cierre del canal pericardioperitoneal durante el

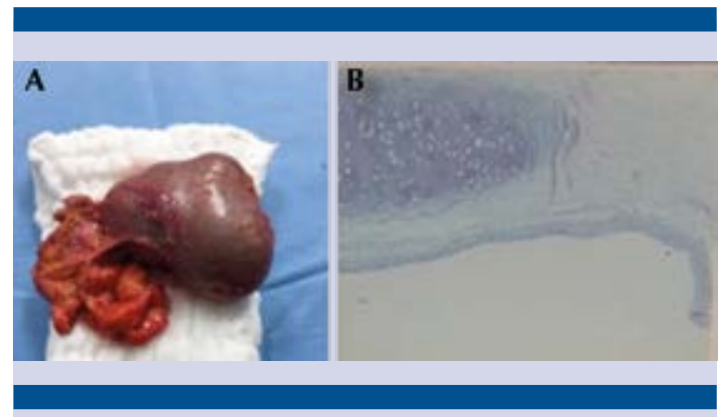

Figura 4. Pieza macroscópica del quiste y la glándula adrenal izquierda (A); citología de la pared del quiste (B). desarrollo embriológico, que provocó la migración de elementos del árbol traqueobronquial a la cavidad abdominal. Otra teoría sugiere que los quistes broncogénicos retroperitoneales pueden considerarse quistes de duplicación de intestino anterior anormalmente diferenciados; esto explica su estrecha relación con órganos derivados del intestino anterior primitivo, como el páncreas o la vesícula biliar. ${ }^{1,4,5}$

Los pacientes con quistes broncogénicos suelen cursar asintomáticos y su diagnóstico se establece de manera fortuita. Nuestro caso padecía, solamente, dolor de espalda inespecífico. Se ha demostrado que estos tumores pueden crecer, sangrar o comprimir estructuras adyacentes y de esta forma considerarlos como síntomas. ${ }^{3-5}$

La resonancia magnética y la tomografía computada son estudios fundamentales para establecer el diagnóstico etiológico de las lesiones. En nuestro caso, la urografía por tomografía computada mostró una imagen hipointensa compatible con una masa sólida retroperitoneal; sin embargo, la resonancia magnética reveló una lesión quística, con líquido de alta proteína, característica de una imagen hiperintensa. ${ }^{5}$

El tratamiento de pacientes con quiste broncogénico retroperitoneal consiste en la extirpación quirúrgica. Aunque la mayoría permanece asintomática, el procedimiento quirúrgico se indica para establecer el diagnóstico, aliviar cualquier síntoma y prevenir complicaciones, especialmente infecciones y posible transformación maligna del quiste. ${ }^{4,5}$

Aunque los quistes broncogénicos son excepcionales, deben considerarse en el diagnóstico diferencial de alguna masa retroperitoneal, particularmente un tumor quístico adyacente a la glándula suprarrenal izquierda, además de proceder con métodos de mínima invasión para el mayor beneficio del paciente. 


\section{REFERENCIAS}

1. Cosío-Pascal M, Cosío-Lima L, Lezama-Urtecho CA, RíosReyna JL. Quiste broncogénico. Reporte de un caso y revisión de la literatura. Neumol Cir Torax 2014;73(1):24-34.

2. Rungea T, Blank A, Stephan C. Schäfer, Candinas D, et al. A retroperitoneal bronchogenic cyst mimicking a pancreatic or adrenal mass. Case Rep Gastroenterol 2013;7:428-432.
3. Dietrich A, Martin de Santiban, Rodriguez-Santos F. Resection of a retroperitoneal bronchogenic cyst: laparoscopic approach. CRSLS MIS Case Reports from SLS.org.

4. Betancourt-Rodríguez V, Archila-López El. Tumores suprarrenales. Revisión bibliográfica. Acta Med Centro 2014;8(1).

5. Haddadin WJ, Reid R, Jind RM. A retroperitoneal bronchogenic cyst: a rare cause of a mass in the adrenal region. $J$ Clin Pathol 2001;54:801-802.

\section{AVISO PARA LOS AUTORES}

Revista Mexicana de Urología tiene una nueva plataforma de gestión para envío de artículos: https://www.revisionporpares.com/index.php/RMUrol ahí podrá inscribirse a la base de datos administrada por el sistema Open Journal System (OJS) que ofrece las siguientes ventajas para los autores:

- Subir sus artículos directamente al sistema.

- Conocer, en cualquier momento, el estado de los artículos enviados, es decir, si ya fueron asignados a un revisor, aceptados con o sin cambios, o rechazados.

- Participar en el proceso editorial corrigiendo y modificando sus artículos hasta su aceptación final. 